\title{
NOUVELLE
}

\section{Amiante et inflammation, rôle de l'inflammasome}

Catherine Dostert, Virginie Pétrilli
Département de Biochimie, Université de Lausanne, Chemin des Boveresses 155, 1066 Epalinges, Suisse.

Catherine.Dostert@unil.ch
> L'amiante comprend un ensemble de substances minérales (silicate de calcium ou de magnésium) de texture fibreuse dont la principale caractéristique est de résister au feu, et de posséder des propriétés isolantes. L'exposition répétée aux fibres d'amiante entraîne communément une fibrose pulmonaire ou asbestose, ainsi que l'apparition de tumeurs spécifiques de la plèvre, du péritoine ou du péricarde, les mésothéliomes. Les mésothéliomes se développent après 30 à 40 ans d'exposition aux poussières d'amiante. L'amiante favorise également l'apparition de tumeurs broncho-pulmonaires, deux à cinq fois plus fréquentes que chez des personnes non exposées, après seulement 10 à 20 ans d'exposition [1].

Des complications similaires sont liées à la silicose, déclenchée par l'inhalation répétée des particules de silice. Dans les deux cas, asbestose et silicose, le développement de la maladie se traduit par une étape initiale d'inflammation.

Bien que de nombreuses études épidémiologiques aient démontré la relation causale entre l'amiante et l'apparition de fibroses pulmonaires et de mésothéliomes, les mécanismes biologiques aboutissant à l'inflammation pulmonaire après exposition à l'amiante ou au silice sont à ce jour très peu connus.

\section{Le récepteur NOD-like Nalp3 \\ des macrophages,} un composant de l'inflammasome Les macrophages pulmonaires jouent un rôle primordial dans l'élimination des pathogènes et des corps étrangers au niveau du poumon en ingérant ces composés pour ensuite les détruire. Le contact qui s'établit entre les macrophages et les corps étrangers active des récepteurs cellulaires de l'immunité innée et induit la sécrétion de médiateurs inflammatoires, dont une cytokine pro-inflammatoire essentielle l'interleukine-1 $\beta$ (IL-1 $\beta$ ). Un certain nombre de récepteurs cellulaires capables de déclencher une réponse inflammatoire sont exprimés par les macrophages, parmi eux les Toll-like receptors (TLR) [11] et les NOD-like receptors (NLR) reconnaissent des signatures moléculaires de pathogènes
(PAMP) ou des signaux de danger (DAMP, damage associated molecular pattern molecules) comme HMGBI (high mobility group box 1), ou encore les protéines S100 ou l'ADN [2] (Figure 1). Un de ces $\mathrm{NLR}$, Nalp3, joue un rôle capital au niveau des macrophages dans la détection d'une variété de PAMP et de DAMP, en induisant notamment la production d'IL-1 $\beta$ [3]. Le récepteur Nalp3 agit au sein d'un complexe moléculaire appelé «l'inflammasome». De façon schématique, ce com-

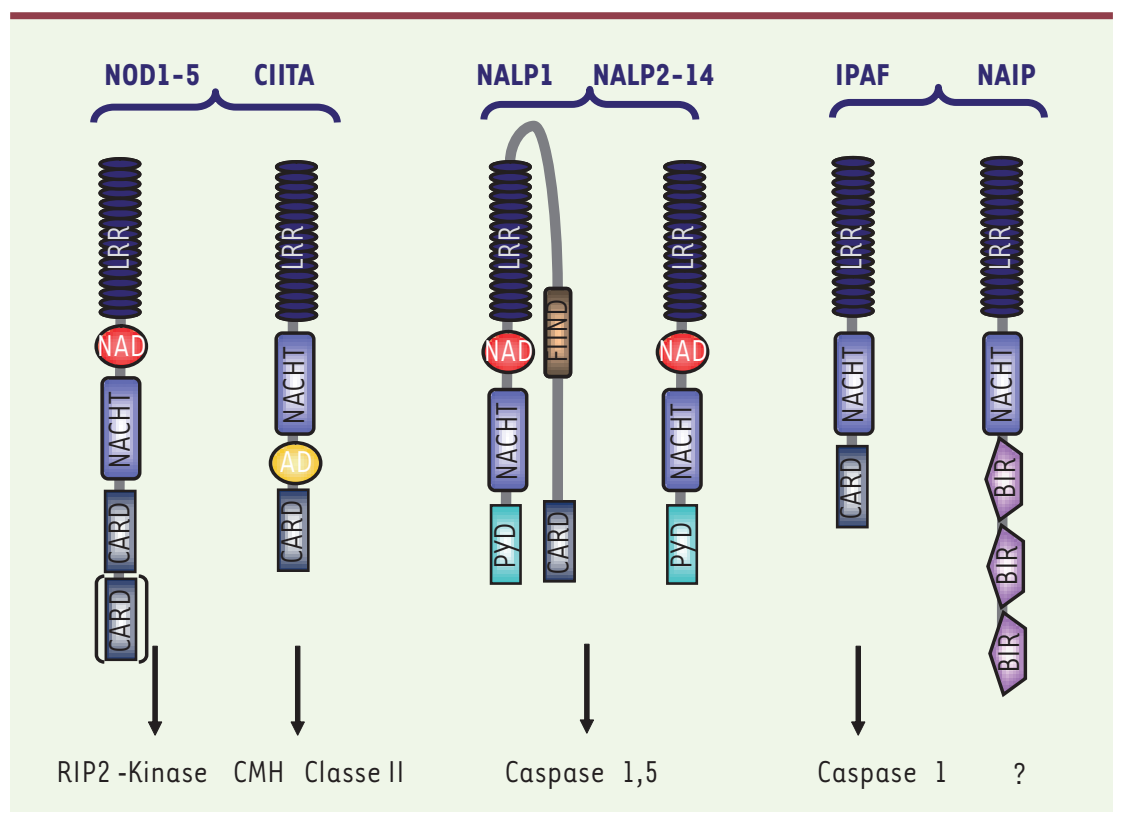

Figure 1. Récepteurs intracellulaires du système immunitaire inné. Les Nod-like receptors (NLR) font partie de la famille des récepteurs intracellulaires de l'immunité innée. La famille des NLR comprend 22 membres dont les NALP (NACHT, LRR and PYD containing protein), les NOD (nucleotide oligomerisation domain), IPAF (ICE protease activating factor), NAIP (neuronal apoptosis inhibitory protein) et le facteur de transcription CIITA. Chaque sous-famille active des voies de signalisation différentes. LRR: leucine-rich repeat; NAD: NACHT associated domain; NACHT: domain present in neuronal apoptosis inhibitory protein (NAIP), the major histocompatibility complex (MHC) class /I transactivator (CIITA), HET- $\varepsilon$ and TPI ; PyD : Pyrin domain ; CARD : caspase recruitment domain; BIR: baculovirus inhibitor of apoptosis (IAP) repeat. 


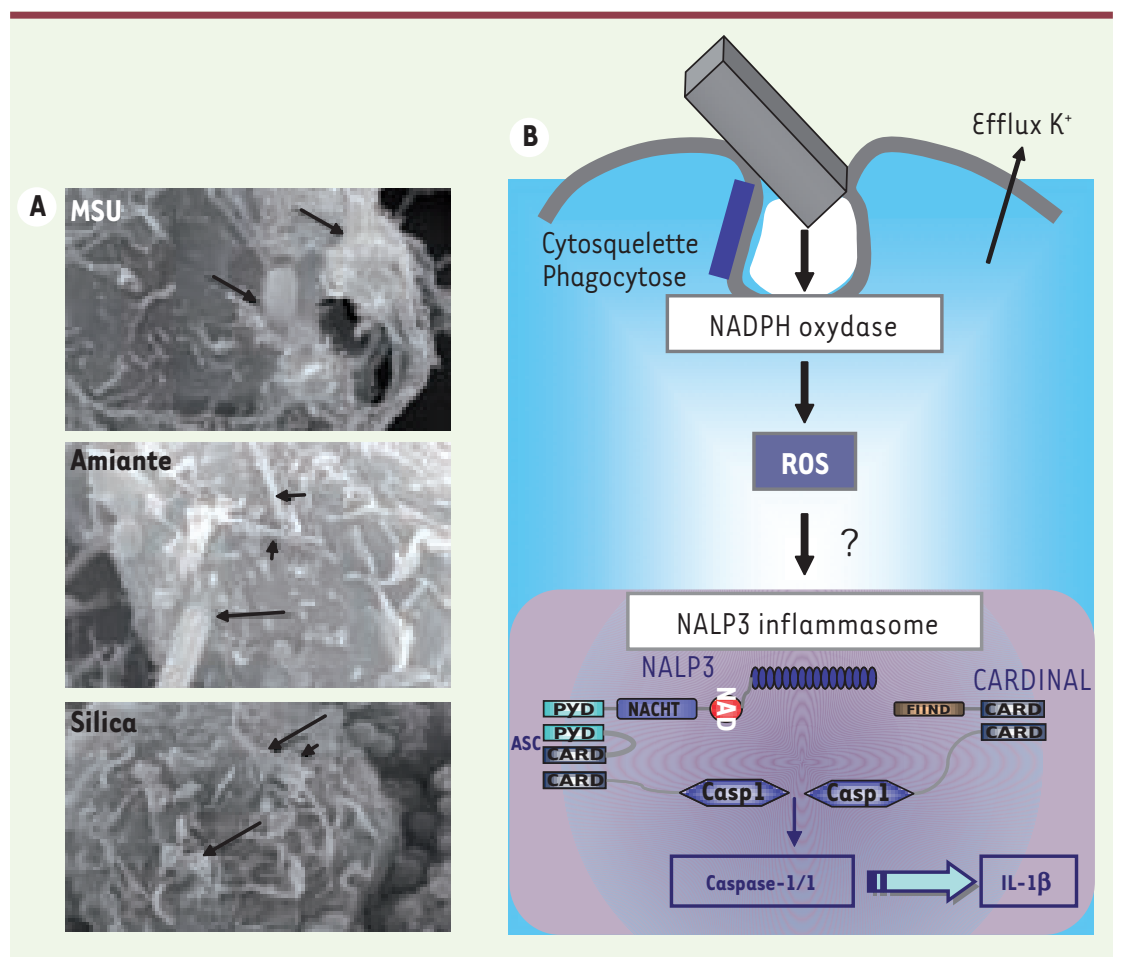

Figure 2. Activation de l'inflammasome Nalp3 par l'amiante. A. Macrophages humains exposés à des particules de MSU, d'amiante ou de silice. Les flèches indiquent les particules entrant dans la cellule. B. L'amiante est phagocytée par les macrophages, induisant l'activation de la NADPH oxydase et la production de dérivés réactifs d'oxygène (ROS). Ces événements activent l'inflammasome Nalp3, ce qui résulte dans la production d'interleukine-1 $\beta$ (IL-1 $\beta$ ) pro-inflammatoire.

plexe est formé par différentes protéines, le récepteur Nalp3, les adaptateurs, ASC et Cardinal, et la caspase-1, enzyme responsable de la maturation du précurseur prolL-1 $\beta$ en IL-1 $\beta$ active [4]. Des mutations de Nalp3 sont associées à trois maladies auto-inflammatoires de transmission autosomique dominante - le syndrome de Muckle Wells ${ }^{1}$, le familial cold auto-inflammatory syndrome (FCAS) et le syndrome chronic infantile neurological cutaneous and articular (CINCA) - qui se caractérisent par des périodes récurrentes de fièvre sans infection associée, accompagnées d'une sécrétion d'IL-1 $\beta$ élevée [5].

\section{La «phagocytose frustrée 》 des fibres d'amiante}

Nalp3 est capable de détecter différents signaux associés à des pathogènes,

1 À ces signes s'associe une surdité neurosensorielle qui s'installe dans l'adolescence. La gravité de l'affection réside dans la survenue inconstante d'une amylose généralisée de type AA (source, orphanet). un phénomène connu sous le nom de «phagocytose frustrée». La déstabilisation du cytosquelette d'actine (responsable de la phagocytose) par la cytochalasine D empêche l'activation de l'inflammasome par l'amiante et la production d'IL-1 $\beta$, démontrant le rôle essentiel de la phagocytose dans l'activation de l'inflammasome Nalp3. L'amiante est également capable d'induire la production des espèces réactives de l'oxygène dans la cellule et le traitement des cellules avec des antioxydants empêche la production d'IL-1 $\beta$ en réponse aux activateurs de l'inflammasome. La source de ces dérivés réactifs de l'oxygène durant la phagocytose pourrait être l'activation d'un complexe membranaire appelé NADPH oxydase, une enzyme activée par la phagocytose des bactéries [12]. L'inhibition pharmacologique de la NADPH oxydase (par le diphénylène iodonium) et son inactivation par réduction de l'expression de la sous-unité p22phox par ARN interférence, diminuent fortement la production d'IL-1 $\beta$ mature en réponse à l'amiante. Enfin, la réduction de l'expression d'une protéine cellulaire impliquée dans la détoxification des dérivés réactifs de l'oxygène, la thiorédoxine, aboutit, à l'inverse, à une production accrue d'IL- $1 \beta$ en réponse à l'amiante et à la silice.

\section{Modèle in vivo}

\section{d'exposition à l'amiante}

Afin d'étudier les conséquences sur l'asbestose de l'inactivation de $\mathrm{Nalp3}$ in vivo, nous avons utilisé un modèle murin d'inhalation de fibres d'amiante. L'exposition à l'amiante de souris de génotype sauvage induit un recrutement de cellules inflammatoires dans les poumons, indiquant une réaction inflammatoire. En revanche, dans les poumons des souris déficientes en Nalp3, ce recrutement de cellules inflammatoires est fortement réduit, de même que la production d'un certain nombre de cytokines, soulignant l'importance de l'inflammasome $\mathrm{Nalp3}$ dans cette pathologie. 
Inhiber l'IL-1, une perspective thérapeutique?

L'ensemble de ces résultats démontrent que la phase inflammatoire induite par l'inhalation de fibres d'amiante est relayée par l'inflammasome Nalp3 et dépendante de la production d'IL-1 $\beta$. Des travaux similaires ont démontré le rôle capital de l'inflammasome dans le développement de la silicose [8]. L'usage d'un inhibiteur naturel de l'IL-1, antagonisant le récepteur de l'IL-1, l'Anakinra, pourrait se révéler extrêmement efficace dans le cadre du traitement de l'asbestose et de la silicose. Cet inhibiteur est déjà utilisé efficacement dans le traitement d'autres maladies associées à Nalp3, comme la goutte [9] et les maladies auto-inflammatoires tel le syndrome de Muckle-Wells [10]. Nalp3 s'avère être une cible intéressante pour le développement de nouveaux médicaments visant à traiter les pathologies inflammatoires. $\diamond$ Asbestos triggers inflammation by activating the Nalp3 inflammasome

\section{RÉFÉRENCES}

1. Mossman BT, Churg A. Mechanisms in the pathogenesis of asbestosis and silicosis. Am J Respir Crit Care Med $1998 ; 157: 1666-80$.

2. Fritz JH, Ferrero RL, Philpott DJ, Girardin SE. Nod-like proteins in immunity, inflammation and disease. Nat Immunol $2006 ; 7: 1250-7$.

3. Petrilli V, Dostert C, Muruve DA, Tschopp J. The inflammasome: a danger sensing complex triggering innate immunity. Curr Opin Immunol 2007 ; 19: 615-22.

4. Martinon F, Burns K, Tschopp J. The inflammasome : a molecular platform triggering activation of inflammatory caspases and processing of prolL-beta. Mol Cell 2002; 10: 417-26.
5. Stojanov S, Kastner DL. Familial autoinflammatory diseases : genetics, pathogenesis and treatment. Curr Opin Rheumatol 2005; 17 : 586-99.

6. Martinon F, Petrilli V, Mayor A, et al. Gout-associated uric acid crystals activate the NALP3 inflammasome. Nature 2006 ; $440: 237-41$.

7. Dostert C, Petrilli V, Van Bruggen R, et al. Innate immune activation through Nalp3 inflammasome sensing of asbestos and silica. Science $2008 ; 320$ : 674-7.

8. Cassel SL, Eisenbarth SC, lyer SS, et al. The Nalp3 inflammasome is essential for the development of silicosis. Proc Natl Acad Sci USA 2008 ; 105 : 9035-40.

9. So A, De Smedt T, Revaz S, Tschopp J. A pilot study of IL-1 inhibition by anakinra in acute gout. Arthritis Res Ther 2007 ; 9 : R28.

10. Hawkins PN, Lachmann HJ, McDermott MF. Interleukin 1-receptor antagonist in the Muckle-Wells syndrome. N Engl J Med 2003 ; 348 : 2583-4.

11. Delneste $Y$, Beauvillain C, Jeannin P. Immunité naturelle: structure et fonction des Toll-like receptors. Med Sci (Paris) 2007 ; $23: 67-73$.

12. Guichard C, Pedruzzi $\varepsilon$, Fay M, et al. Les Nox/Duox: une nouvelle famille de NADPH oxydases. Med Sci (Paris) $2006 ; 22: 953-9$.

\section{NOUVELLe}

\section{Le génome de l'ornithorynque À la croisée des chemins}

Simone Gilgenkrantz

Médecine/Sciences, 9, rue Basse, 54330 Clérey-sur-Brénon, France. simone.gilgenkrantz@club-internet.fr
> L'ornithorynque (Ornithonryncus anatinus) qui - comme son nom l'indique - a pour nez un bec de canard, est un animal si improbable qu'à la fin du XVIII ${ }^{e}$ siècle, les zoologistes anglais hésitèrent à croire à son existence [1]. En effet, admettre qu'un mammifère à fourrure de castor puisse avoir un bec et des pattes de canard, qu'il ponde des œufs, mais qu'une fois éclos, la femelle les allaite et que le mâle adulte soit venimeux grâce à un ergot situé sur les pattes postérieures nécessitait une certaine crédulité. Mais les observations faites en Australie confirmèrent qu'il s'agissait bien d'un monotrème (pourvu d'un cloaque comme les oiseaux et les reptiles) et qu'il était le seul survivant de la famille des Ornithorynchidae, pourtant jadis importante si l'on en juge par les espèces fossiles retrouvées.

\section{Biologie des monotrèmes}

Animal semi-aquatique, nocturne et très craintif, il vit dans les cours d'eau des régions froides d'Australie et de Tasmanie. II peut rester des heures entières à des températures basses (autour de $5^{\circ} \mathrm{C}$ ) car bien qu'il soit homéotherme, sa fourrure doublée de tissu adipeux lui permet de

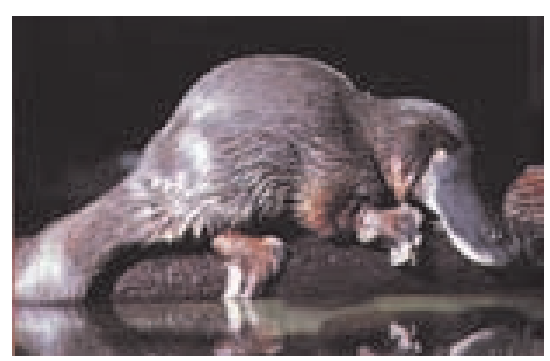

maintenir sa température à $31^{\circ} \mathrm{C}$. On le trouve aussi dans la réserve d'Healesville (État du Victoria) où il est plus facile de l'observer, mais il se reproduit mal en captivité. Situés à l'embranchement entre mammifères et reptiles, l'ornithorynque et les échinidés (à l'aspect de gros hérissons, dont il ne reste plus que quelques espèces) forment le petit groupe des monotrèmes. Ceux-ci se sont séparés des thériens (marsupiaux et euthériens) il y a environ 166 millions d'années (Figure 1). Ainsi, bien qu'il soit un mammifère, cet animal possède des caractères reptiliens ancestraux tels que l'oviparité et l'aspect des spermatozoïdes.

La femelle pond un à trois œufs par an. Plus sphériques que les œufs d'oiseaux, ils sont incubés pendant une dizaine de jours. Il est possible que, dans l'évolution, la sécrétion lactée ait précédé l'apparition des mammifères et qu'elle ait exercé sur les œufs parcheminés des synapsides pendant l'incubation un rôle protecteur, empêchant la 